\title{
Assessment of Urban Aerial Taxi with Cryogenic Components under Design Environment for Novel Vertical Lift Vehicles (DELIVER)
}

\author{
Christopher.A.Snyder@nasa.gov \\ Propulsion Systems Analysis Branch \\ NASA John H. Glenn Research Center
}

ATIO.TFPC-04. Transformational Aircraft Technologies

AIAA Aviation 2017 Forum

Denver, Colorado

June 6, 2017 


\section{Motivation}

DELIVER vision: "Can we bring 100 years of aeronautics knowledge to the new entrepreneurs' desktop with a design environment for emerging vertical lift vehicles?"

Present tools and methods do a pretty good job for today's vehicles and missions. However, there is increasing interest in new classes / types of vertical-lift vehicles, improving mobility and overall utility / flexibility. Advanced electric propulsion and power systems offer the potential for reduced noise and point of use emissions, enhanced vehicle and mission capability, etc.

Assess the capabilities of methods and tools to capture these new vehicles and propulsion / power technologies. 


\section{Outline}

- Introduce baseline vehicle and hybrid systems studied

- Review propulsion and energy systems

- Vehicle sizing and mission analysis tool

- Genset sizing and mission profiles

- Results

- Mission power levels

- Maximum mission range and number of on-demand mobility (ODM) missions, also recharge / refuel

- Thermal loads, cooling airflow requirements

- Summary

- Next Steps 


\section{Baseline vehicle and propulsion}

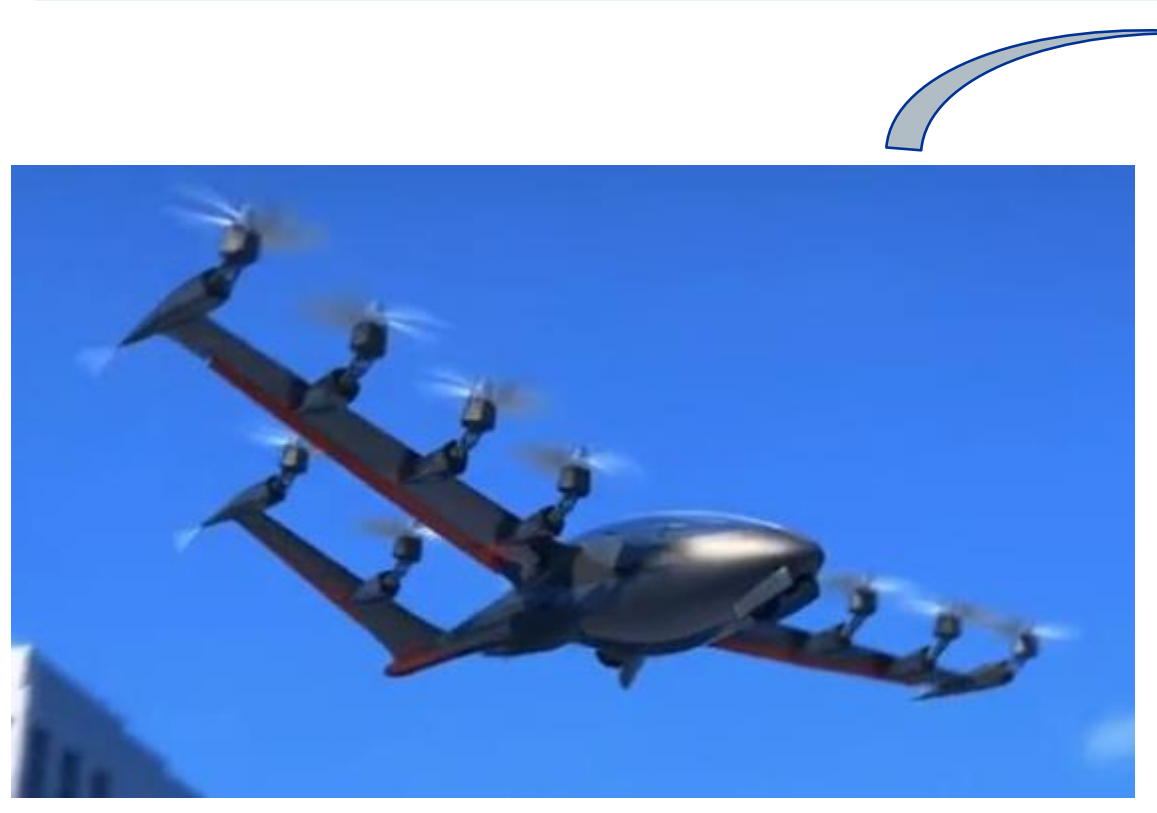

Urban aerial taxi (VTOL-ops) $450 \mathrm{lb}$. of passengers / luggage / cargo

$+200 \mathrm{lb}$. pilot
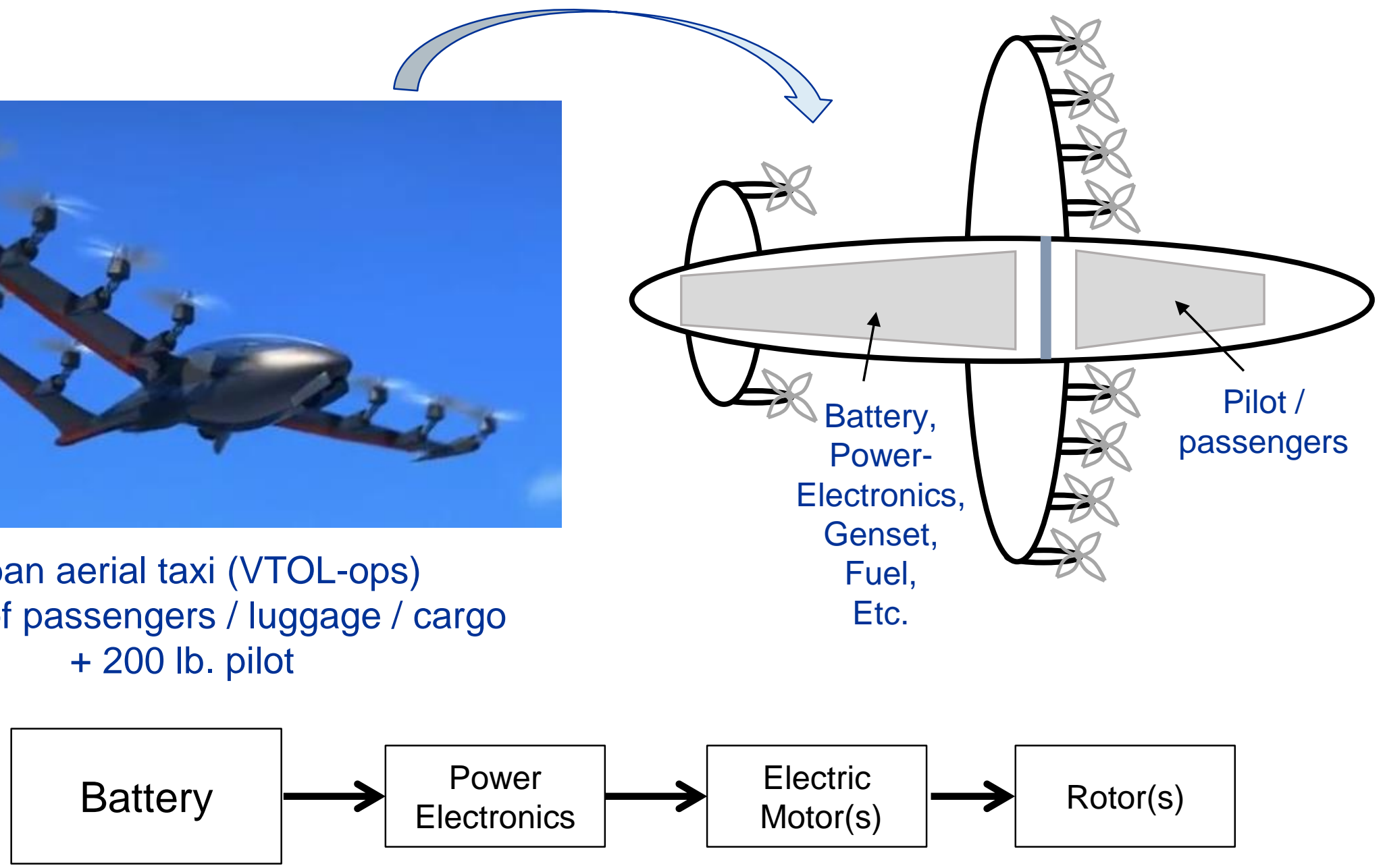

Baseline propulsion system 


\section{Baseline vehicle and hybrid propulsion}
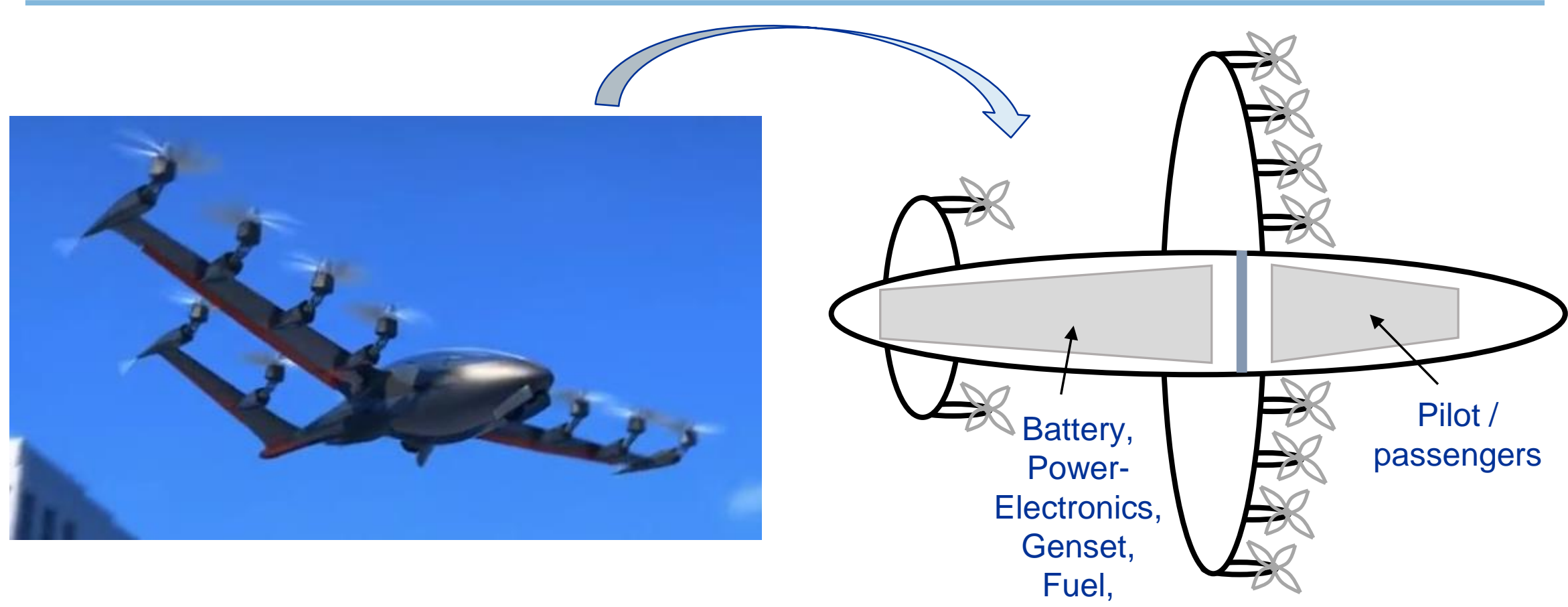

Etc.

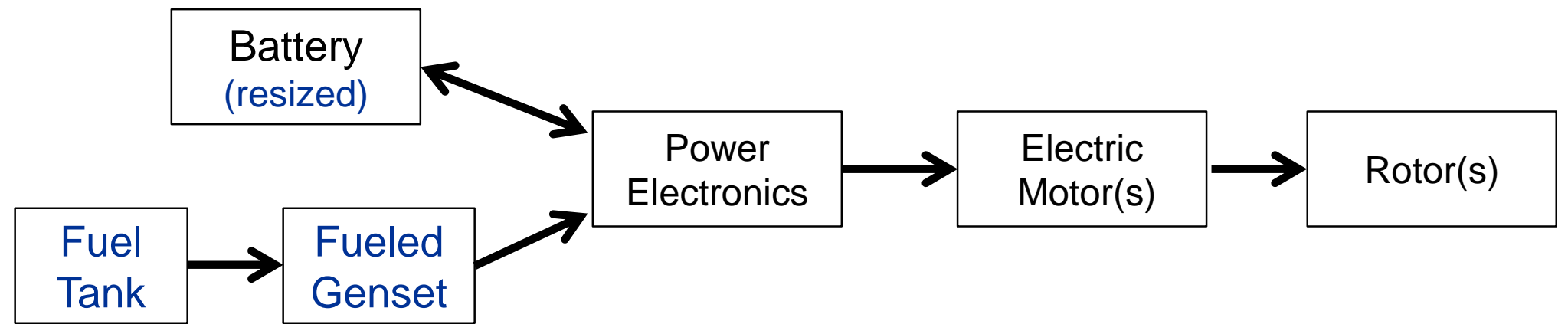

Hybrid propulsion system 


\section{Baseline vehicle and LNG hybrid propulsion}
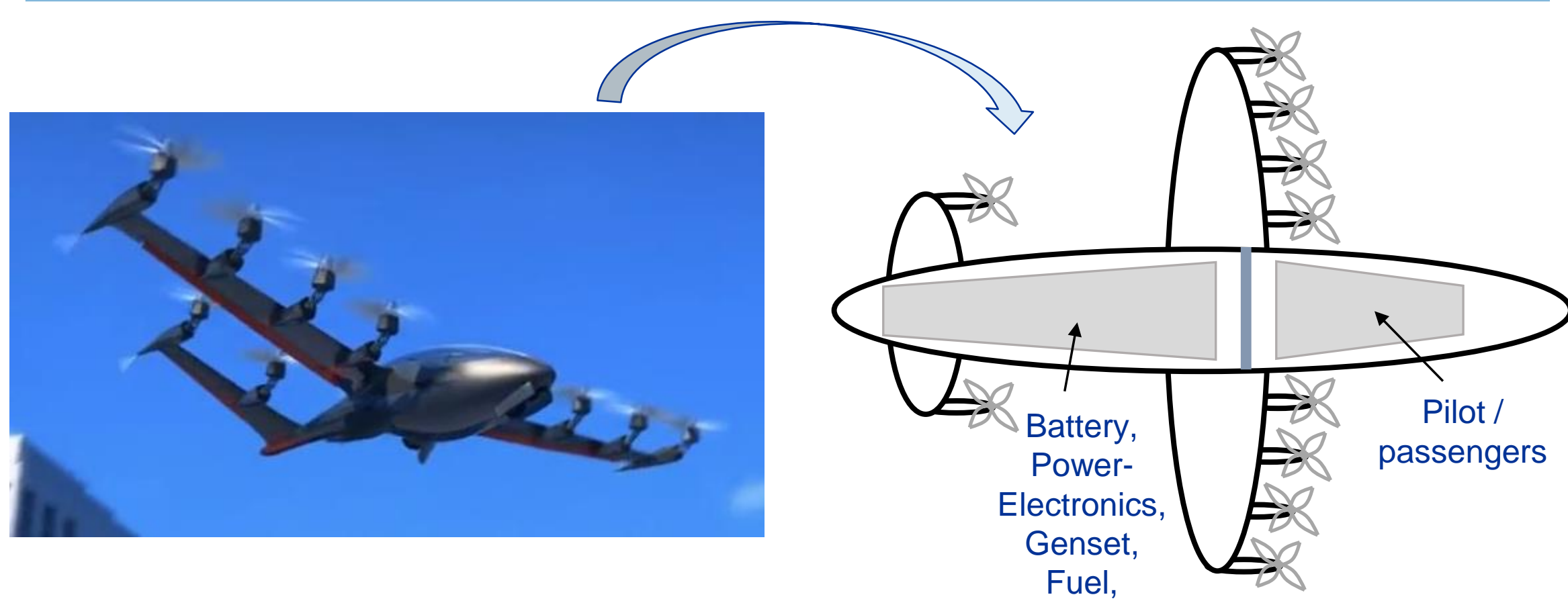

Etc.

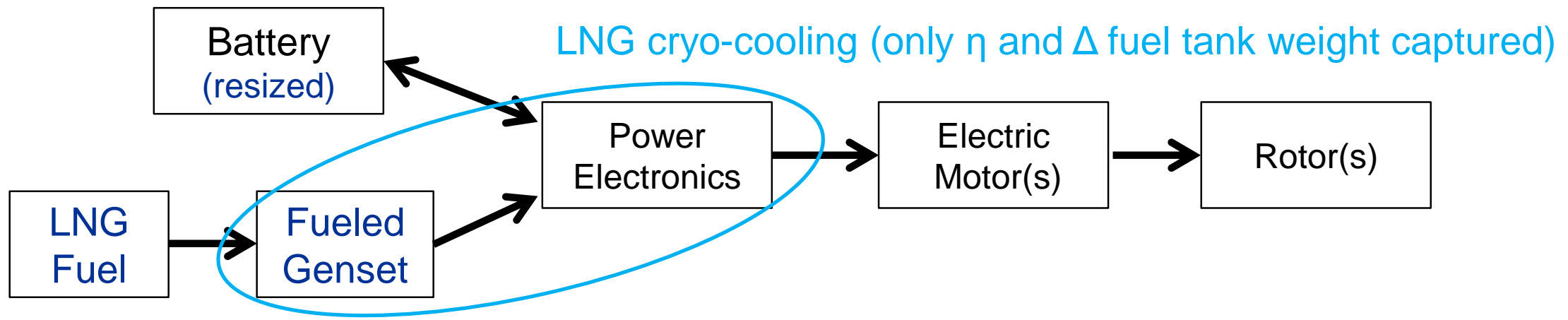

Liquid Natural Gas (LNG) Hybrid propulsion system 


\section{Impressive weight and loss reductions for Electric Motor / Electronics are occurring}

\begin{tabular}{|c|c|c|c|c|c|}
\hline $\begin{array}{c}\text { Technology } \\
\text { year }\end{array}$ & $\begin{array}{c}\text { Power/weight, } \\
\text { hp/lb. (kW/kg) }\end{array}$ & $\begin{array}{c}\text { Electric } \\
\text { motor } \eta, \%\end{array}$ & $\begin{array}{c}\text { Power } \\
\text { Electronics } \\
\eta, \%\end{array}$ & $\begin{array}{c}\text { Net } \eta, \\
\%\end{array}$ & $\begin{array}{c}\text { Net } \\
\text { Loss, \% }\end{array}$ \\
\hline State of the art & $\mathbf{1 . 9 ( 3 . 1 )}$ & 90 & 94 & 85 & 15 \\
\hline 15 year & $3.4(5.6)$ & 95 & 98 & 93 & 7 \\
\hline 30 year & $\mathbf{4 . 9 ( 8 . 0 )}$ & 98 & 99 & 97 & 3 \\
\hline $\begin{array}{l}\text { Non-cryogenic } \\
\text { Power-to-weight includes electric motor (3,8,16 hp/lb.) + power electronics }(5,6,7 \mathrm{hp} / \mathrm{lb} .)\end{array}$
\end{tabular}

"Some 15 year" goals are being demonstrated today Reduced losses = less thermal management (less weight)

* Dever, T.P.; Duffy, K.P.; Provenza, A.J.; Loyselle, P.L.; Choi, B.B.; Morrison, C.R.; and Lowe, A.M. "Assessment of Technologies for Noncryogenic Hybrid Electric Propulsion", NASA TP-2015-216588, January 2015. 
Electric motors significantly better than fueled systems in efficiency and power to weight

BUT efficiency doesn't overcome deficiencies in energy storage (critical for range / endurance)

\begin{tabular}{|c|c|c|c|c|}
\hline Engine type & $\begin{array}{c}\text { Power / } \\
\text { weight, hp/lb. } \\
(\mathrm{kW} / \mathrm{kg})\end{array}$ & $\eta, \%$ & $\begin{array}{c}\text { Fuel, energy } \\
\text { density, MJ/kg } \\
(\mathrm{Wh} / \mathrm{kg})\end{array}$ & $\begin{array}{c}\text { Net energy } \\
\text { density, MJ/kg } \\
(\mathrm{Wh} / \mathrm{kg})\end{array}$ \\
\hline all-electric, SOA & $1.9(3.1)$ & 85 & $0.70(194)$ & $0.60(165)$ \\
\hline 15 year & $3.4(5.6)$ & 93 & $1.75(486)$ & $1.63(450)$ \\
\hline 30 year & $4.9(8.0)$ & 97 & 3.15 (875) & $3.06(850)$ \\
\hline $\begin{array}{c}\text { Diesel cycle, SOA } \\
15 \text { year } \\
30 \text { year }\end{array}$ & $\begin{array}{l}0.53(0.9) \\
1.06(1.8) \\
1.59(2.7)\end{array}$ & 37 & $\begin{array}{c}\text { Diesel, } \\
43.0(12,000)\end{array}$ & $15.9(4,400)$ \\
\hline
\end{tabular}

For electric systems, "Fuel" is lithium battery, cell only average of lithium ion and sulfur technologies Electric system power to weight for electric motor reported at 3,8 , and $16 \mathrm{hp} / \mathrm{lb}$. and power electronics at 5, 6, and $7 \mathrm{hp} / \mathrm{lb}$. for state of the art (SOA), 15 and 30 year technology assumptions (from Dever).

Only 15 year technology levels reported for this study 


\section{Analysis Tool: NASA Design and Analysis of Rotorcraft (NDARC)}

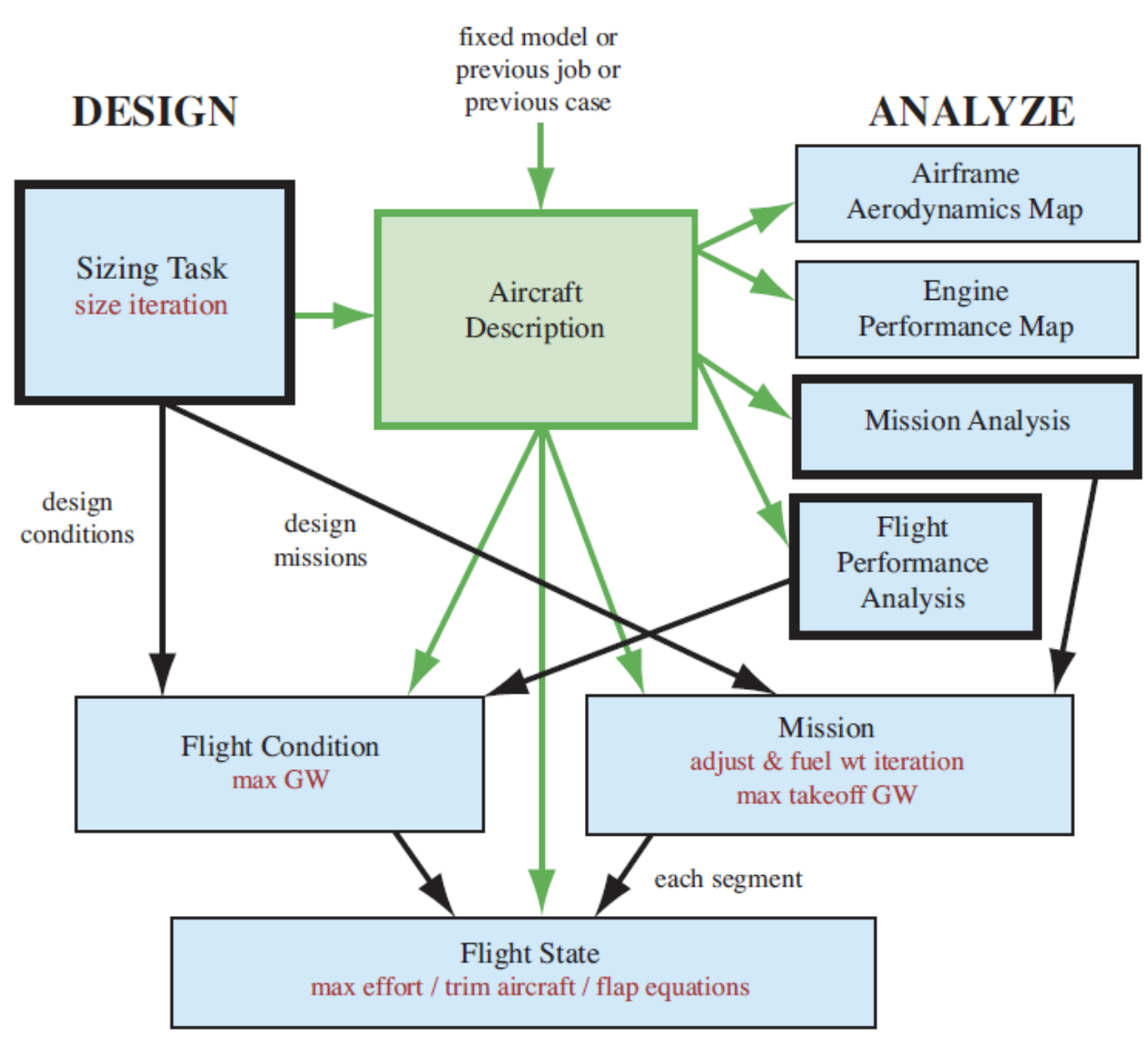

Overall Program Layout

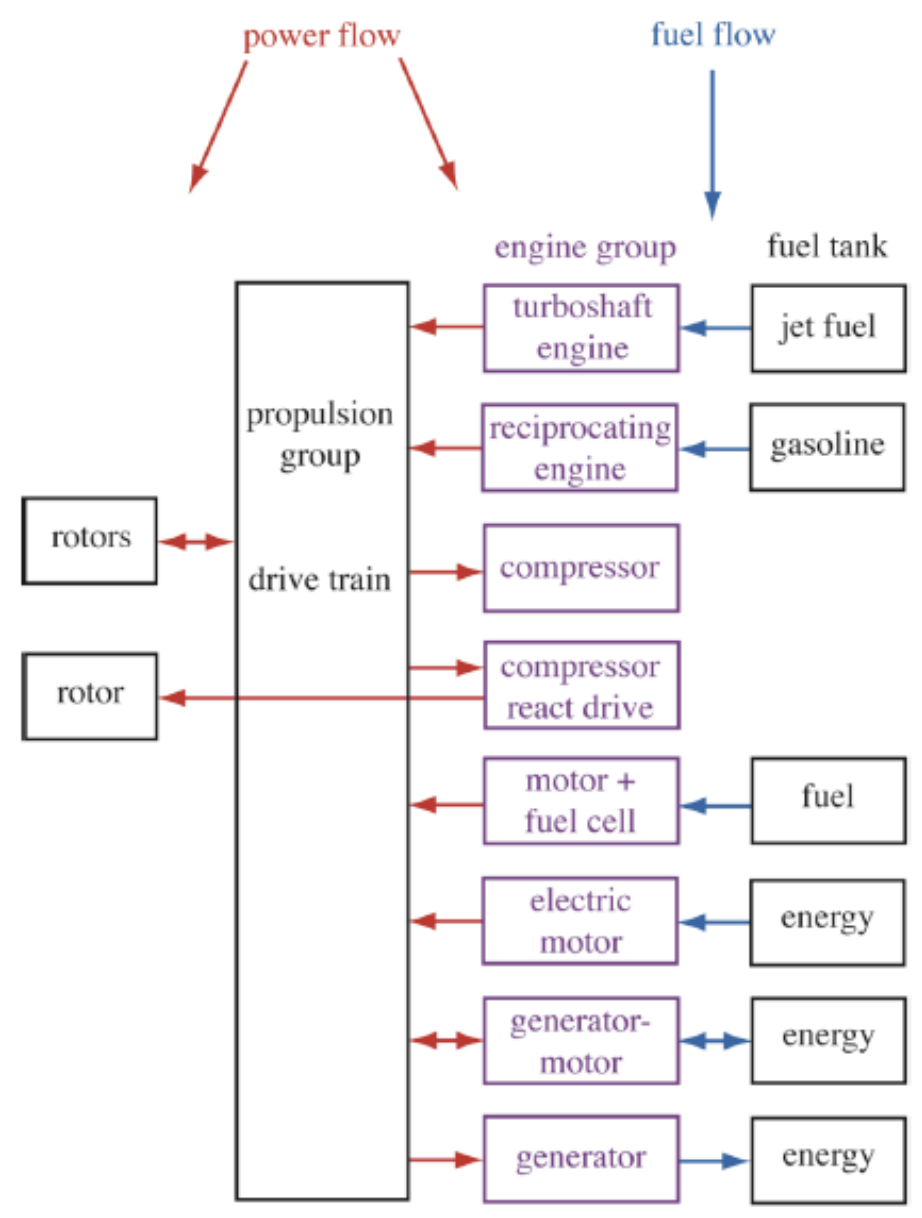

Propulsion / energy models 


\section{Aircraft / Genset Power and Mission Profile}

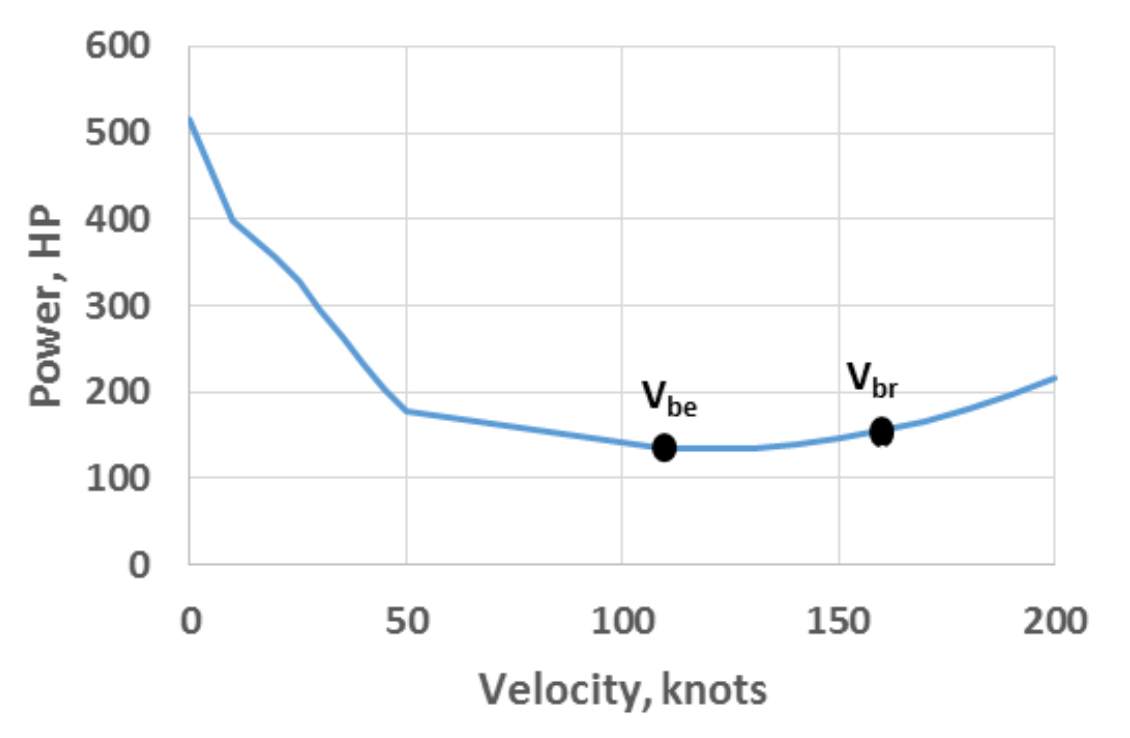

Fueled genset sized at 150 , 175 , and $200 \mathrm{hp}$ (engine hp, not generated electric power)

\section{Vehicle sizing (150 nmi) maximum range and ODM (20 or $50 \mathrm{nmi})$ mission profile}

1) 5 min. idle, Takeoff + 2 min. hover (OGE)

2) Climb to cruise at maximum power, range credited

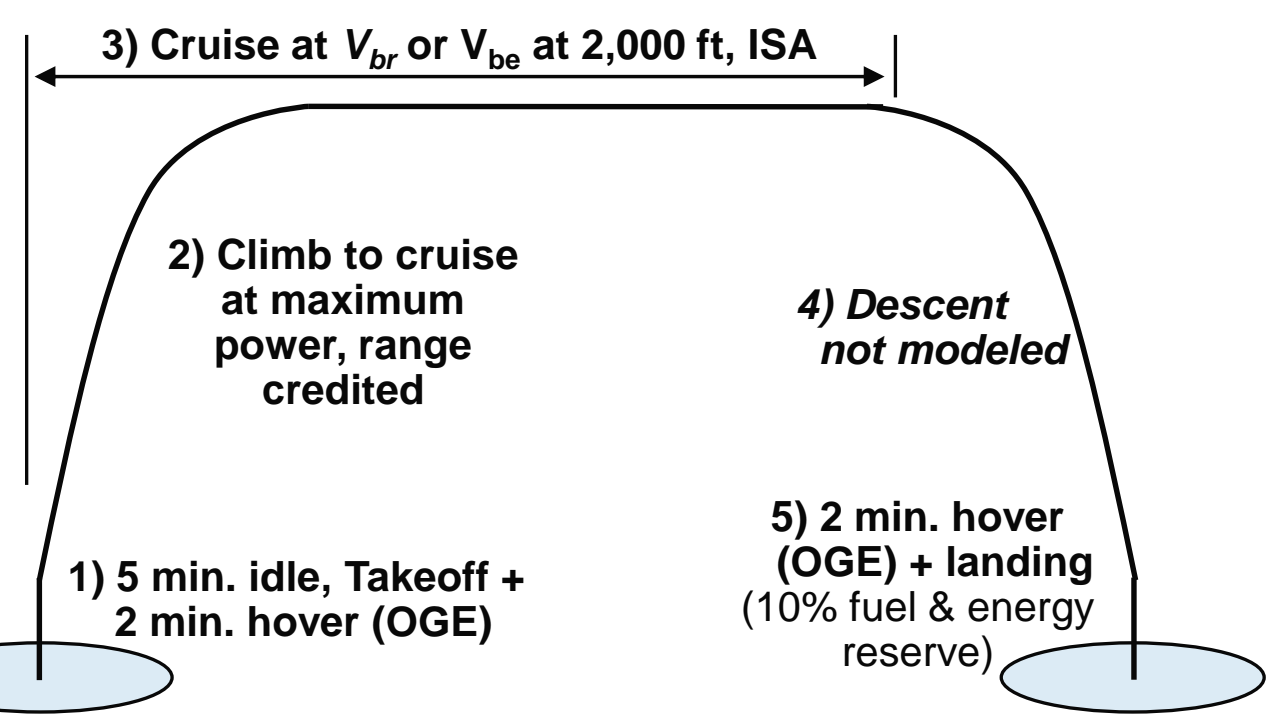

ODM = on demand mobility OGE = out of ground effect 


\section{Results}




\section{Power requirements over the mission}

Max power requirement set by hover

Cruise power levels significantly less

Climb power level can be lower (only limited to hover power)

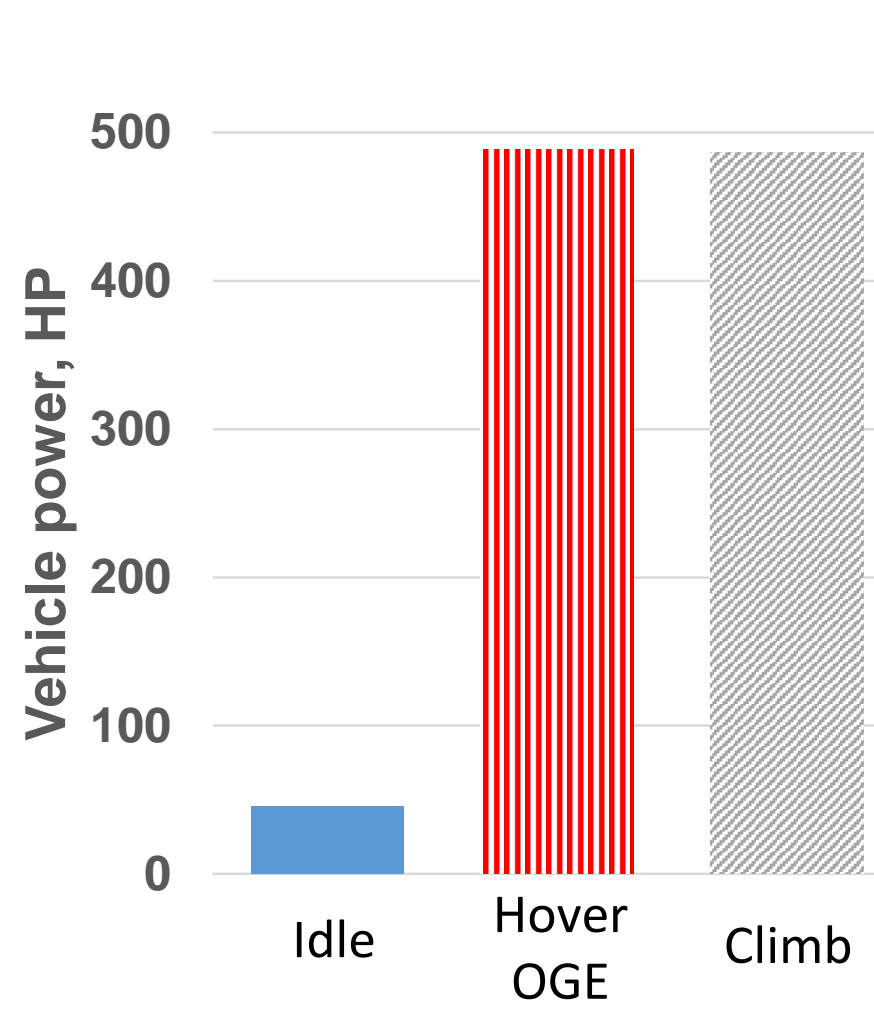




\section{Vehicle Parameters}

\begin{tabular}{|c|c|c|c|}
\hline Parameter $\downarrow$ & All-Electric \\
Baseline & Vehicle & $\begin{array}{c}150 \mathrm{hp} \\
\text { conventional } \\
\text { cooled hybrid }\end{array}$ & $\begin{array}{c}200 \text { hp cryo- } \\
\text { cooling assisted } \\
\text { hybrid }\end{array}$ \\
\hline Design gross weight (DGW), lb. (kg) & $3,676(1,671)$ & $3,678(1,672)$ & $3,673(1,669)$ \\
\hline Empty weight, lb. (kg) & $3,021(1,373)$ & $2,813(1,279)$ & $2,788(1,267)$ \\
\hline Nominal fuel weight, lb. (kg), \% DGW & 0 & $210(95), 6 \%$ & $230(105), 6 \%$ \\
\hline Battery + BMS weight, lb. (kg), \% DGW & $919(418), 25 \%$ & $498(226), 13.5 \%$ & $437(199), 12 \%$ \\
\hline Genset Weight, lb. (kg), \% DGW & 0 & $211(96), 6 \%$ & $256(116), 7 \%$ \\
\hline Fuel volume, gallon, (I) & 0 & $30.7(116)$ & $89.1(337)$ \\
\hline Battery volume, gallon, (I) & $80.4(304)$ & $43.6(165)$ & $38.3(145)$ \\
\hline Fuel Energy, MJ & 0 & 4,096 & 4,695 \\
\hline Battery energy, MJ & 609 & 330 & 290 \\
\hline
\end{tabular}

- 15 year technology: Genset size+fuel trades well for weight and energy versus battery (1.75 MJ/kg, 486 w-h/kg)

- Will have to check LNG fuel tank volume 


\section{Hybrid system significantly improves max range}

\begin{tabular}{|c|c|c|c|c|c|c|c|}
\hline Vehicle $\rightarrow$ & Baseline & \multicolumn{3}{c|}{$\begin{array}{c}\text { Conventionally-cooled } \\
\text { hybrid }\end{array}$} & \multicolumn{4}{c|}{$\begin{array}{c}\text { Cryogenically cooled } \\
\text { hybrid }\end{array}$} \\
\hline & & $150 \mathrm{hp}$ & $175 \mathrm{hp}$ & $200 \mathrm{hp}$ & $150 \mathrm{hp}$ & $175 \mathrm{hp}$ & $200 \mathrm{hp}$ \\
\hline \multicolumn{8}{|c|}{ Maximum range missions } \\
\hline All $\mathrm{V}_{\mathrm{br}}, \mathrm{nmi}$ & 150 & $298^{*}$ & $460^{*}$ & 496 & $378^{*}$ & $530^{*}$ & 580 \\
\hline $\begin{array}{c}\text { Mix of } \mathrm{V}_{\mathrm{br}} \text { and } \mathrm{V}_{\text {be }}, \\
\mathrm{nmi}\end{array}$ & $\begin{array}{c}122 \\
\left(\text { all } \mathrm{V}_{\mathrm{be}}\right.\end{array}$ & 470 & 492 & & 554 & 575 & \\
\hline
\end{tabular}

* Battery energy limited range

Hybrid systems increased max range by 2 to 4 times (hydrocarbon energy density)

Larger genset improved range (reduced battery weight, more fuel, more mission at best range speed) 
Hybrid system enables more ODM missions (before recharge / refuel)

\begin{tabular}{|c|c|c|c|c|c|c|c|c|}
\hline Vehicle $\rightarrow$ & $\begin{array}{c}\text { Base } \\
\text { line }\end{array}$ & \multicolumn{3}{|c|}{$\begin{array}{c}\text { Conventionally-cooled } \\
\text { hybrid }\end{array}$} & \multicolumn{3}{c|}{ Cryogenically cooled hybrid } \\
\hline & & $150 \mathrm{hp}$ & $175 \mathrm{hp}$ & $200 \mathrm{hp}$ & $150 \mathrm{hp}$ & $175 \mathrm{hp}$ & $200 \mathrm{hp}$ \\
\hline \multicolumn{7}{|c|}{ Multiple ODM missions } \\
\hline $\begin{array}{c}\text { Number of } 20 \mathrm{nmi} \\
\text { missions (17 min.) }\end{array}$ & 3 & 6.5 & 6.9 & 7.3 & 7.8 & 8 & 8 \\
\hline Hold time, minutes $\dagger$ & $7 \ddagger$ & 30 & 21 & 15 & 27 & 18.5 & 13 \\
\hline $\begin{array}{c}\text { Number of } 50 \mathrm{nmi} \\
\text { missions (28 min.) }\end{array}$ & 2 & 4 & 4.6 & 4.9 & 5.3 & 5.6 & 7 \\
\hline Hold time, minutes $\dagger$ & $10 \ddagger$ & 36 & 22 & 15 & 31 & 19 & 12 \\
\hline
\end{tabular}

$\dagger$ Time on ground between ODM missions to self-recharge battery to full

‡ No self-recharge capability, 3C / 500 kW charger required (cooling?)

Hybrid systems significantly increased number of possible ODM missions before recharge / refuel.

Hybrid genset self-recharge takes longer than "fastest" electric recharge 


\section{Thermal requirements (heat load, kw)}

\section{Hover}

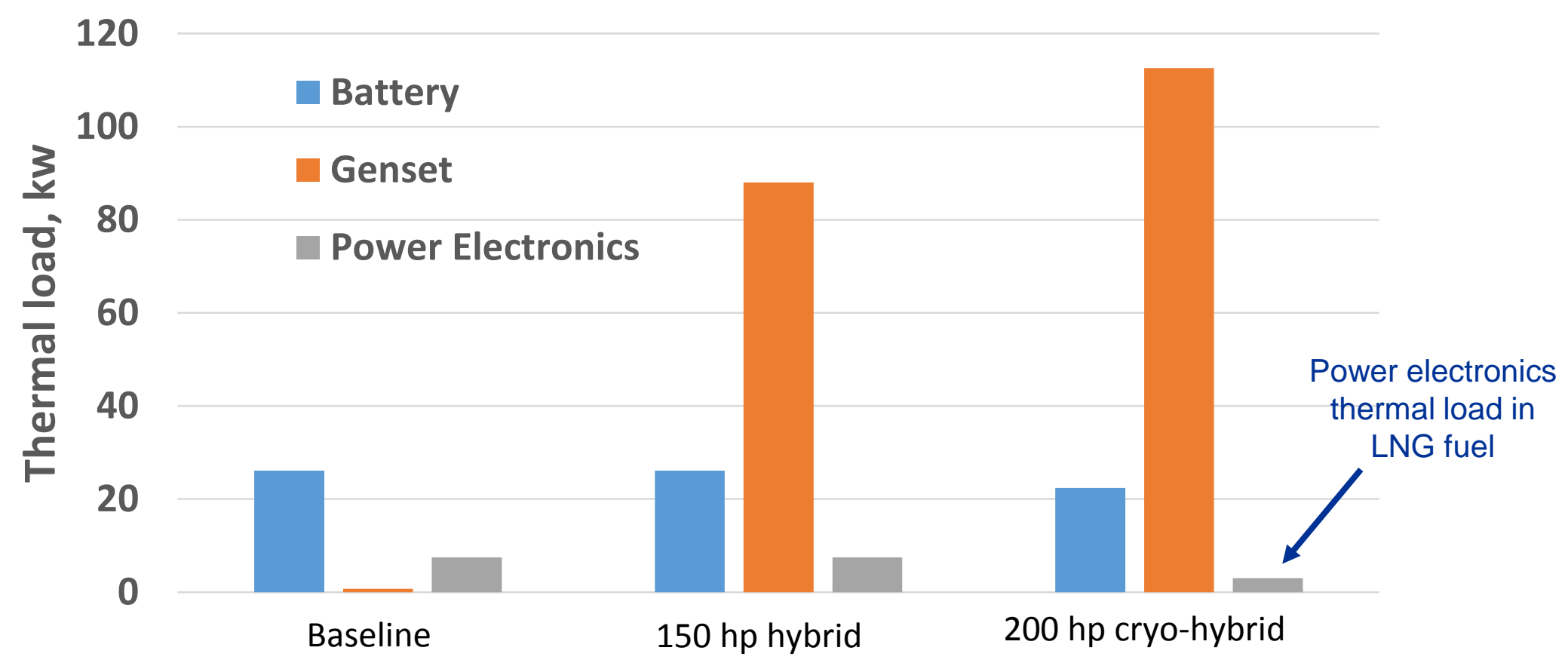

Hybrid genset thermal loads dominate (where present)

Battery thermal loads similar over different configurations

Power electronics load fairly minimal, cryo cooling reduces it further 


\section{Thermal requirements (alt $=0$, ISA and alt $=5 \mathrm{k}-\mathrm{ft}, \mathrm{ISA}+20^{\circ} \mathrm{C} / 36^{\circ} \mathrm{F}$ )}

Hover cooling airflow, $\mathrm{ft}^{\wedge} 3 /$ minute)

alt=0, ISA

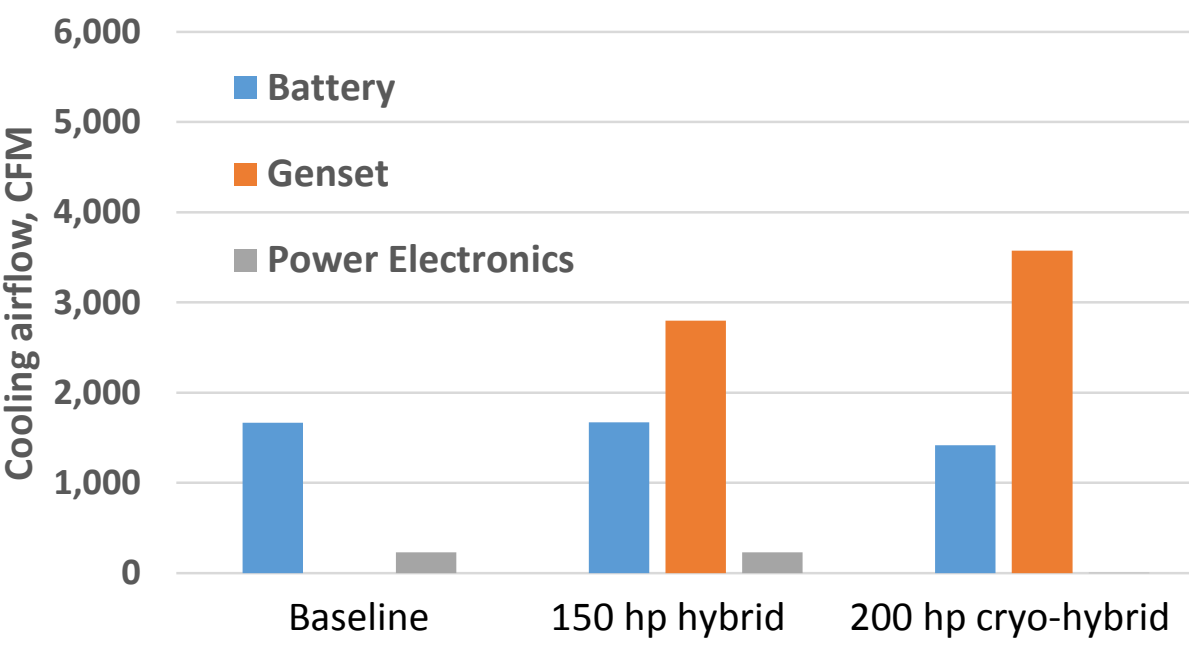

alt $=5 \mathrm{k}-\mathrm{ft}, \mathrm{ISA}+20^{\circ} \mathrm{C} / 36^{\circ} \mathrm{F}$

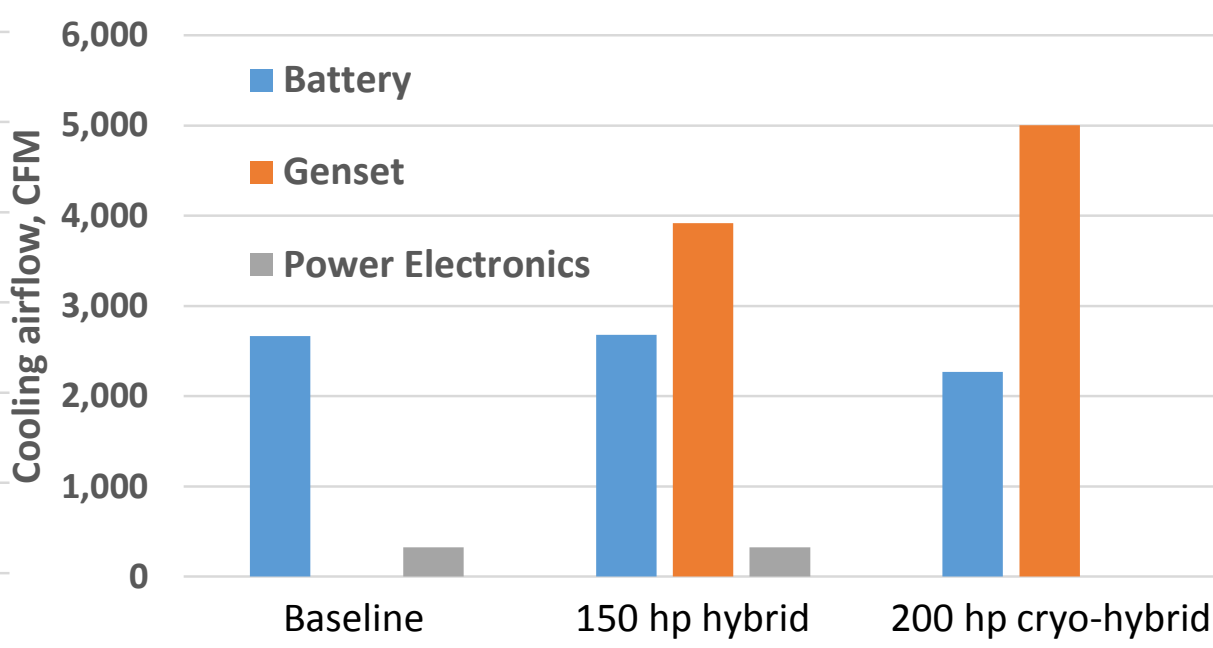

$5 \mathrm{k}$-ft altitude, hot day increases volumetric airflow rate

Genset cooling flows still dominate

Greater airflow increase for battery, lower maximum use temperature

Climb results similar (similar power levels, compensating effects of temperature and density) 


\section{Thermal requirements (heat load, kw)}

\section{Cruise}

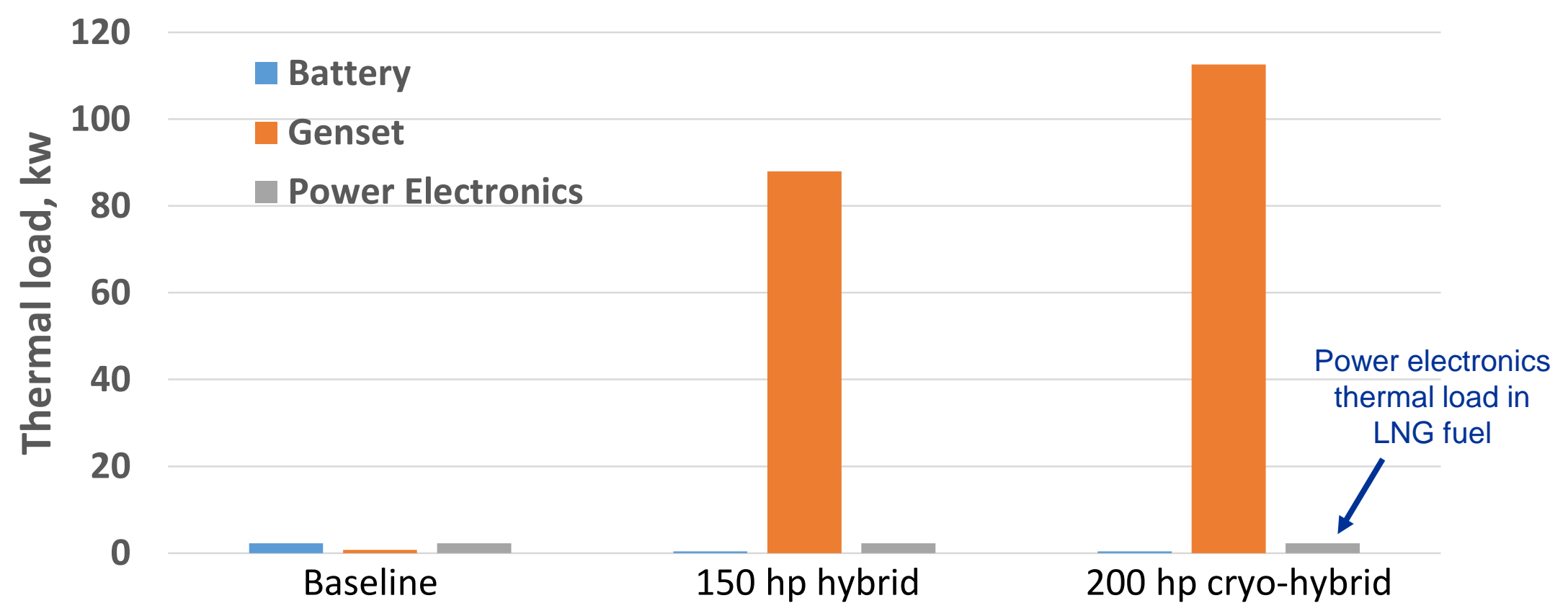

Hybrid genset thermal loads dominate (where present)

Battery thermal load is $\alpha I^{2} R$, drops by order of magnitude from hover / climb

Battery and power electronic thermal loads fairly minimal 


\section{Thermal requirements (alt $=0$, ISA and alt $=5 \mathrm{k}-\mathrm{ft}, \mathrm{ISA}+20^{\circ} \mathrm{C} / 36^{\circ} \mathrm{F}$ )}

Cruise cooling airflow, $\mathrm{ft}^{\wedge} 3 /$ minute)

5,000

alt=2k-ft, ISA

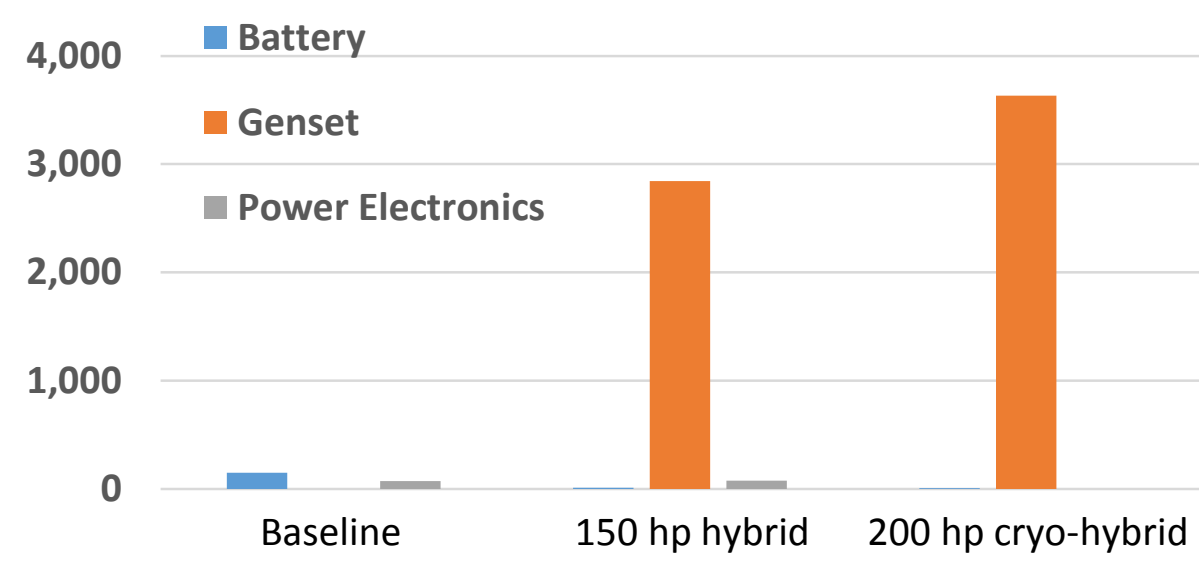

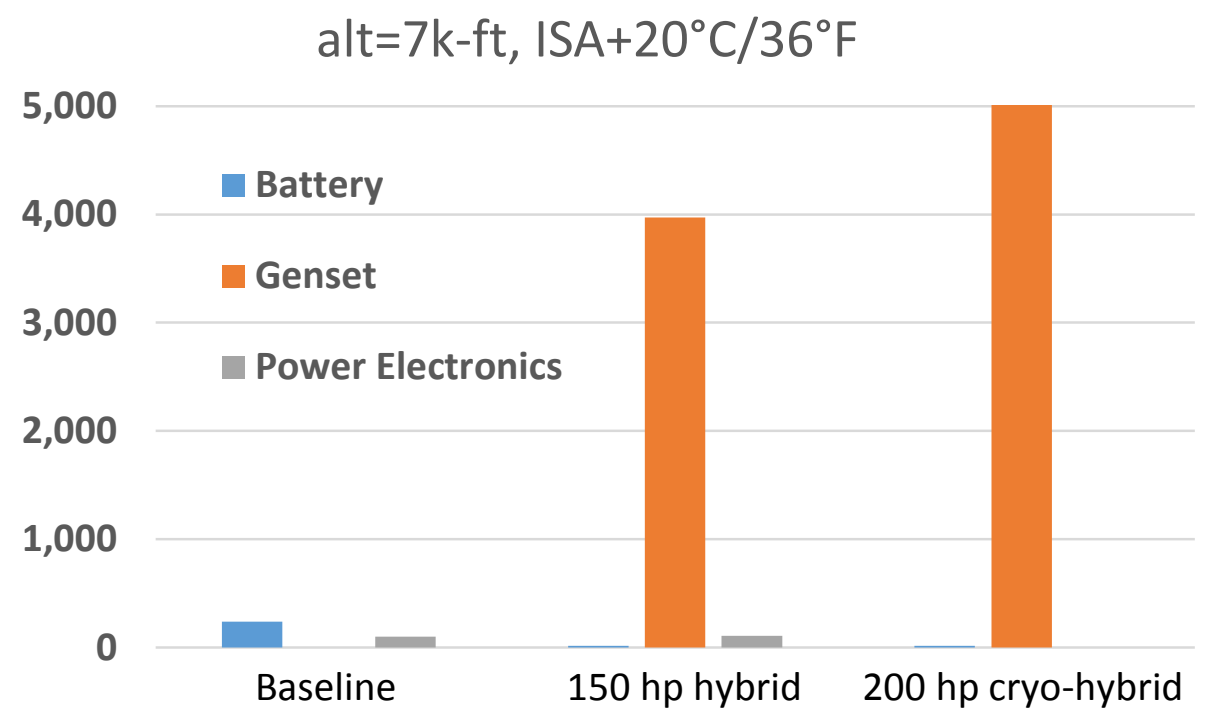

$5 \mathrm{k}$-ft altitude gain and hot day increases volumetric airflow rate

Genset cooling flow still dominates, minimal change from hover / climb results because genset operation at constant power (turbocharged diesel)

Battery airflow change similar to previous, but much smaller totals (battery discharge rate is $<1 \mathrm{C}$ ), order of magnitude smaller than hover / climb 


\section{Summary}

- Hybrid systems with energy-dense hydrocarbon fuels can significantly increase maximum range or number of shorter range missions vehicle can achieve. (Genset size, figure of merit?)

- Vehicles with hybrid system can self-recharge battery, but requires more time than all-electric (infrastructure)

- LNG cryo-cooling improves performance (even before further design efforts for weight, volume, drag of cooling systems - TBD)

- Hybrid systems generate a lot of waste heat that must be removed

- Battery and power electronics heat seems to be hover / climb dominated. Although fast charging is another high-heat condition. (Active cooling or design / infrastructure consideration.)

- NDARC design tools appears capable of modeling vehicle, mission and systems, although need to develop propulsion and power systems models outside of NDARC. 


\section{Next Steps}

- Perform more detailed layout / analysis for conventionally and LNG cryo-cooled gensets.

- Check if bulk sizing parameters capture real system considerations (more / how detailed)

- Develop validated models (although data may be lacking)

- Document process, methods, etc. for others to use (and build upon). DELIVER ends with FY17, unsure who might pick up work, Revolutionary Vertical Lift Technology project?

- Other cycles? (Fuel cells, recuperative gas turbines, ?)

- Further tool development

- Methods / tools to automate the process

- Include in NDARC directly or through new OpenMDAO framework (also in development) 


\section{Acknowledgments}

This work was supported by the Aeronautics Research Mission Directorate (ARMD)

Transformative Aeronautics Concepts Program (TACP) / Convergent Aeronautics Solutions (CAS) Project / Design Environment for Novel Vertical Lift Vehicles (DELIVER) Sub-Project.

Advanced Air Vehicle Program (AAVP) / Revolutionary Vertical Lift Technology (RVLT) Project 


\section{Questions?}





\section{Backup Slides}




\section{Vehicle Parameters}

\begin{tabular}{|c|c|c|c|}
\hline Parameter $\downarrow$ & All-Electric \\
Baseline & $\begin{array}{c}150 \mathrm{hp} \\
\text { conventional } \\
\text { cooled hybrid }\end{array}$ & $\begin{array}{c}200 \text { hp cryo- } \\
\text { cooling assisted } \\
\text { hybrid }\end{array}$ \\
\hline Design gross weight (DGW), lb. (kg) & $3,676(1,671)$ & $3,678(1,672)$ & $3,673(1,669)$ \\
\hline Disk loading / wing loading, lb./ft^2 & $10 / 50$ & $10 / 50$ & $10 / 50$ \\
\hline Empty weight, lb. (kg) & $3,021(1,373)$ & $2,813(1,279)$ & $2,788(1,267)$ \\
\hline Nominal fuel weight, lb. (kg), \%DGW & 0 & $210(95), 6 \%$ & $230(105), 6 \%$ \\
\hline Battery+BMS weight, lb. (kg), \%DGW & $919(418), 25 \%$ & $498(226), 13.5 \%$ & $437(199), 12 \%$ \\
\hline Genset Weight, lb. (kg), \%DGW & 0 & $211(96), 6 \%$ & $256(116), 7 \%$ \\
\hline Fuel volume, gallon, (I) & 0 & $30.7(116)$ & $89.1(337)$ \\
\hline Battery volume, gallon, (I) & $80.4(304)$ & $43.6(165)$ & $38.3(145)$ \\
\hline Fuel Energy, MJ & 0 & 4,096 & 4,695 \\
\hline Battery energy, MJ & 609 & 330 & 290 \\
\hline Sea level max rated power, hp (kW) & $578(431)$ & $578(431)$ & $578(431)$ \\
\hline $\begin{array}{c}\text { Propulsion engines and power } \\
\text { electronics weight, lb. (kg), \%DGW }\end{array}$ & $307.3(140), 8 \%$ & $310(141), 8 \%$ & $312(141), 8 \%$ \\
\hline
\end{tabular}




\section{Rotor area variation $\rightarrow$ hover efficiency / effectiveness (\& hover power)}

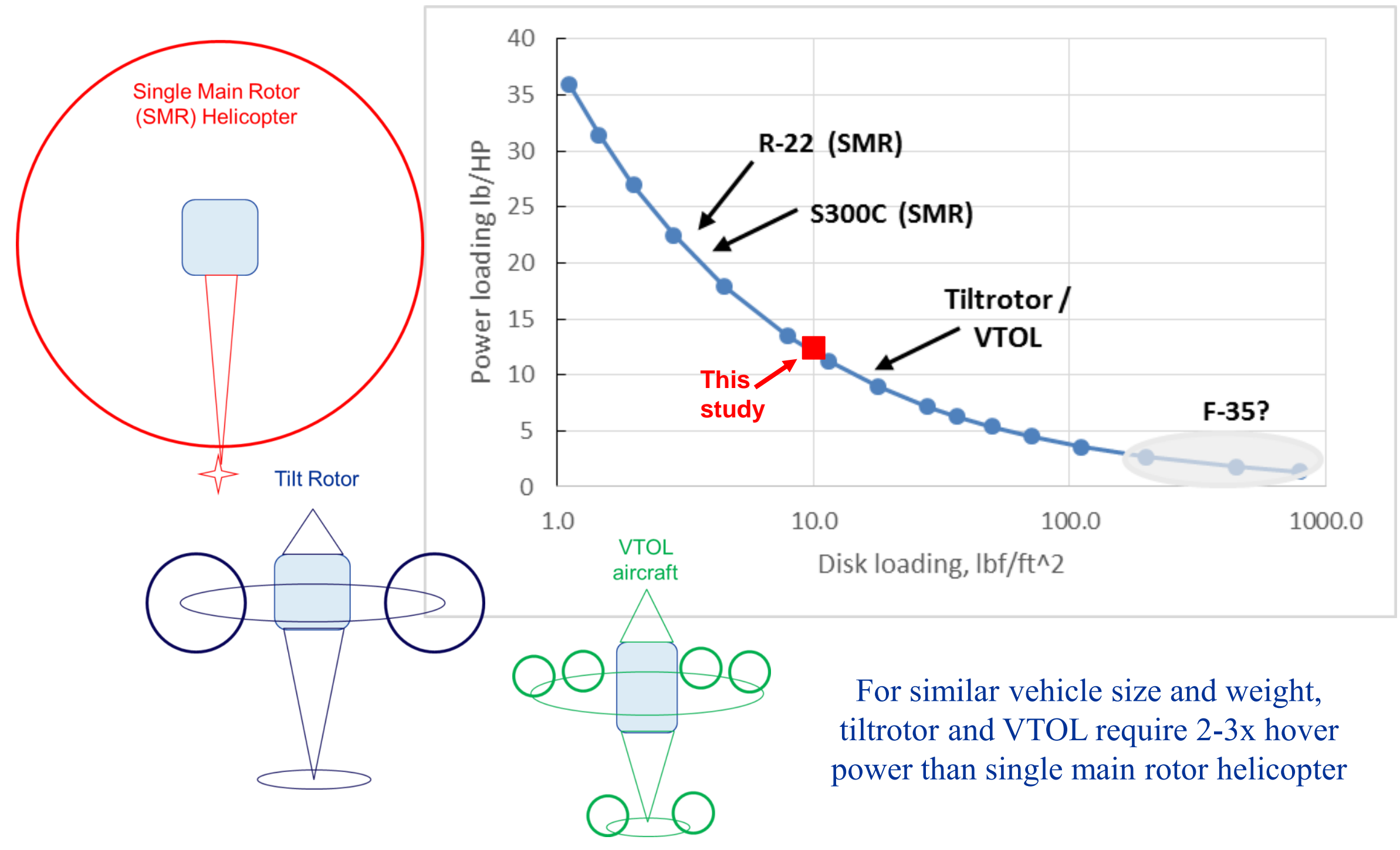


